Review

\title{
Flux-Vortex Pinning and Neutron Star Evolution
}

\author{
M. ALI ALPAR \\ Faculty of Engineering and Natural Sciences, Sabancı University, 34956, Istanbul, Turkey. \\ E-mail: alpar@sabanciuniv.edu
}

MS received 22 May 2017; accepted 24 July 2017; published online 12 September 2017

\begin{abstract}
G. Srinivasan et al. (1990) proposed a simple and elegant explanation for the reduction of the neutron star magnetic dipole moment during binary evolution leading to low mass X-ray binaries and eventually to millisecond pulsars: Quantized vortex lines in the neutron star core superfluid will pin against the quantized flux lines of the proton superconductor. As the neutron star spins down in the wind accretion phase of binary evolution, outward motion of vortex lines will reduce the dipole magnetic moment in proportion to the rotation rate. The presence of a toroidal array of flux lines makes this mechanism inevitable and independent of the angle between the rotation and magnetic axes. The incompressibility of the flux-line array (Abrikosov lattice) determines the epoch when the mechanism will be effective throughout the neutron star. Flux vortex pinning will not be effective during the initial young radio pulsar phase. It will, however, be effective and reduce the dipole moment in proportion with the rotation rate during the epoch of spindown by wind accretion as proposed by Srinivasan $e t$ al. The mechanism operates also in the presence of vortex creep.
\end{abstract}

Keywords. Neutron stars—evolution-magnetic fields.

\section{Introduction}

Two of G. Srinivasan's seminal contributions have been of particular interest to the author. The first was the paper with Radhakrishnan immediately after the discovery of the first millisecond pulsar PSR B 1937+21 (Backer et al. 1982). Immediately after the discovery two groups independently explained millisecond pulsars as the result of accretion in low mass X-ray binaries (LMXBs) (Alpar et al. 1982; Radhakrishnan $\&$ Srinivasan 1982). Both groups made the bold inference that the LMXB magnetic fields must be as weak as $10^{8}-10^{9} \mathrm{G}$ in order to lead to millisecond equilibrium rotation rates, and predicted that the period derivative $\dot{P}$ of the millisecond radio pulsar would be as low as $10^{-19} \mathrm{~s} \mathrm{~s}^{-1}$. This prediction was soon verified by the measurement of $\dot{P}=1.2 \times 10^{-19} \mathrm{~s} \mathrm{~s}^{-1}$ from PSR B 1937+21 (Backer et al. 1983) indicating $10^{8}-10^{9} \mathrm{G}$ fields in millisecond pulsars.

Alpar (2008) reviewed the arguments of these papers on the 10th anniversary of the eventual discovery of the first accreting millisecond X-ray pulsar (AMXP; Wijnands and van der Klis 1998). Alpar et al. (1982) started from the expectation that neutron stars in LMXB have weak magnetic fields. Millisecond periods would be attained as the equilibrium periods with typical LMXB accretion rates $\dot{M} \sim 0.1 \dot{M}_{\text {Edd }}$ if the dipole magnetic field of the neutron star were $B \sim 10^{9} \mathrm{G}$. At the end of accretion a millisecond pulsar would emerge with a period derivative $\dot{P} \sim 10^{-19} \mathrm{~s} \mathrm{~s}^{-1}$, on the 'spin-up' or 'birth' line in the $P \dot{P}$ diagram, shown for the first time in our paper. Radhakrishnan \& Srinivasan (1982) started their argument by noting the lack of a supernova remnant, or any X-ray emission, from a nebula powered by the pulsar. If the millisecond pulsar had a conventional $10^{12} \mathrm{G}$ magnetic field it would be very young, and would be associated with a supernova remnant and a pulsar wind nebula. Using the observational upper limits on the $\mathrm{X}$-ray luminosity of the source they deduced empirically that the dipole magnetic field must be less than about $4 \times 10^{8} \mathrm{G}$ and $\dot{P}$ must be less than about $10^{-19} \mathrm{~s} \mathrm{~s}^{-1}$. They then noted that such a weak magnetic field would yield spin-up to a millisecond rotation period as the equilibrium period after accretion in a binary system. Our two groups independently arrived at the same picture tracing the available clues in different orders.

After the discovery of the first accreting millisecond X-ray pulsar, the LMXB SAX 1808.43658 by Wijnands \& van der Klis (1998), others were discovered, including some that make transitions 
between X-ray and radio epochs (Papitto et al. 2013). The connection between LMXB/AMXP and millisecond radio pulsars is now firmly established. Two basic questions arise regarding the evolution of these systems: (i) how come some millisecond pulsars are now no longer in binaries? (ii) how come the magnetic fields of the millisecond pulsars and LMXB/AMXP are so weak compared to the magnetic fields of young neutron stars? To answer (i), there are established evolutionary scenarios that explain the demise of the companion and emergence of a single radio pulsar after the LMXB phase. Regarding the second question, one class of explanations for the low magnetic fields of millisecond pulsars invoke the burial of the magnetic field under accreted material during the LMXB phase. This is somewhat conjecturally dependent on the accretion history.

The second paper by G. Srinivasan et al. (1990) gives an elegant and convincing answer to the question: Why are the dipole magnetic fields of neutron stars in LMXB $\sim 10^{-3}$ times weaker than the fields in young radio pulsars? Rotation powered pulsars seem to retain initial dipole magnetic fields of $\sim 10^{12} \mathrm{G}$ throughout their active pulsar lifetimes of $\sim 10^{6}-10^{7}$ years. What happens to a neutron star in a binary during the subsequent $10^{8}-10^{9}$ yr lifetime of evolution culminating in the LMXB phase to cause such a reduction of the dipole moment? Srinivasan et al. (1990) proposed that this field reduction is due to the pinning of quantized vortex lines in the neutron star core superfluid to the flux lines in the proton superconductor.

The neutron superfluid in the core of the neutron star takes part in the spindown by sustaining a flow of quantized vortex lines in the direction away from the rotation axis. The protons in the core are expected to be in the Type II superconducting phase (Baym et al. 1969). Due to the pinning between vortex lines and the flux lines of the Type II proton superconductor in the neutron star, core flux lines would be carried outward by the vortex lines. Thus flux would be expelled as the neutron star spins down under external torques. Spindown would induce a reduction in the dipole magnetic field in the same proportion as the reduction in the rotation rate:

$\frac{B(t)}{B(0)}=\frac{\Omega(t)}{\Omega(0)}$.

This depends on the core being entirely in a phase of superfluid neutrons coexisting with Type II superconducting protons. If parts of the core contain normal matter or Type I superconducting protons, these parts might relax their magnetic fields on shorter timescales, as the magnetic field in the crust regions is expected to do, and the long-term evolution of the dipole surface field could still be governed by the SBMT mechanism, equation (1). In any case we will assume with SBMT, that Type II superconductivity and flux-vortex pinning are indeed the dominant features governing the magnetic field evolution of the neutron star.

In the earlier epoch of binary evolution preceding the LMXB phase, the neutron star spins down while accreting from the stellar wind of its companion star to periods $P \sim 100-1000$ s seen in high mass X-ray binaries with wind accretion, like Vela $X-1$. Wind spindown from typical pulsar periods $P \sim 0.1$ s to these periods would cause a reduction in the dipole magnetic field by a factor of $\sim 1000$, yielding $B \sim 10^{9} \mathrm{G}$ at the start of the LMXB epoch. In section 2, a comment on flux line-vortex line pinning and creep against this pinning, with emphasis on the effects of toroidally oriented flux lines has been made. In section 3, the application of the scenario with spindown by wind accretion is discussed.

\section{Flux-vortex pinning}

The possibility of flux line-vortex line pinning was briefly noted by Muslimov and Tsygan (1985). Sauls (1989) pointed out the importance of this for neutron star dynamics in his lecture in the 1988 NATO ASI on 'Timing Neutron Stars'. In his 1989 review on 'Pulsars: Their Origin and Evolution', Srinivasan underlined the importance of this coupling not only for explaining field decay in the evolution leading to millisecond pulsars, but also for explaining why the field does not decay all the way to zero but has the typical value $B \sim 10^{9}$ $\mathrm{G}$ in the old population (Srinivasan 1989). He noted that an interesting possibility is implicit in the paper by Muslimov and Tsygan (1985), although not exploited by them. There are two sets of vortices in the quantum fluid interior: the magnetic vortices (flux lines) referred to above, and the vortices in the neutron superfluid. Muslimov and Tsygan (1985) suggested that the flux lines could get pinned to the normal cores of the superfluid vortices. Srinivasan et al. (1990) were the first to discuss in detail the evolutionary importance of this coupling in their seminal paper.

For a poloidal orientation of flux lines, there are easy directions along which the motion of vortex lines away from the rotation axis, required for the spin-down of the neutron superfluid, will proceed without encountering pinning against flux lines. In other directions, vortex motion will have to encounter flux lines and proceed via creep over the flux vortex pinning junctions by thermal activation. This problem of vortex creep over 
poloidal flux lines was addressed by Sidery and Alpar (2009). As is generally true for magnetohydrodynamic stability, the flux line distribution in neutron stars is likely to include a toroidal component. Topologically unavoidable vortex pinning and creep against toroidal flux lines was addressed by Gügercinoğlu \& Alpar (2014) and by Gügercinoğlu (2017).

The magnetic dynamics of the Type II superconductor in coupling with the rotational dynamics of the neutron superfluid is a complicated problem for which the detailed solution on all different timescales is not known (Passamonti et al. 2017). The essentials relevant for the evolutionary scenario of Srinivasan et al. (1990) were presented in an important paper by Ruderman et al. (1998). I will try to review and clarify the arguments of this paper, which develops a criterion for vortex-flux line pinning to effectively lead to flux decay induced by spindown, as proposed by Srinivasan et al. (1990).

Ruderman et al. (1998) started by noting that the other forces sustaining currents on a magnetic system should balance the Lorentz force on a macroscopic volume, yielding

$\mathbf{F}=\frac{\mathbf{J} \times \mathbf{B}}{\mathbf{c}}$.

With $\mathbf{J}=\sigma \mathbf{E}$ and $\mathbf{E}=-(\mathbf{v} \times \mathbf{B}) / \mathbf{c}$, one obtains the relation

$v \cong \frac{F c^{2}}{\sigma B^{2}}$

between the relative velocity $v$ of charges and magnetic flux, the force per unit volume $F$, the conductivity $\sigma$ and the magnetic field $B$. For the Type II superconductor, they replace the magnetic field with macroscopic average $\langle B\rangle=n_{\Phi} \Phi_{0}$, where $n_{\Phi}$ is the area density of flux lines and $\Phi_{0}=h c / 2 e$ is the flux quantum, to obtain

$v_{\Phi} \cong \frac{F c^{2}}{\sigma_{\mathrm{eff}}\left(n_{\Phi} \Phi_{0}\right)^{2}}$

for the relative speed of the flux lines with respect to the charges (the center of mass of the electron - superconducting proton plasma), in terms of the effective conductivity $\sigma_{\text {eff }}$. The drag force due to the scattering of electrons from the magnetic field in the cores of the flux lines drives the decay of the magnetic flux by the flow of the flux lines at speed $v_{\Phi}$ away from the magnetic axis (or the circular axis of the toroidal distribution in the case of toroidal flux lines). This force (per unit length of flux line) is a linear drag force

$\mathbf{f}=-\eta\left(v_{\mathrm{c}}-v_{\mathrm{L}}\right)$

where $v_{\mathrm{c}}$ and $v_{\mathrm{L}}$ are the velocities of the electrons and the flux line respectively. The coefficient $\eta$ has been calculated for scattering of electrons from a magnetized neutron vortex line (Alpar et al. 1984a), and can readily be scaled for the scattering of electrons from a flux line. This drag force will govern the motion of the flux line with respect to the proton superfluid/superconductor, of velocity $v_{\mathrm{s}}^{\mathrm{p}}$, through the Magnus equation, or equivalently, the flux line moves with respect to the background proton superfluid such that the drag force balances the Lorentz force on the flux line:

$\mathbf{f}=\frac{n_{\mathrm{p}} c}{e} \boldsymbol{\Phi}_{0} \times\left(v_{\mathrm{s}}^{\mathrm{p}}-v_{\mathrm{L}}\right)=\rho_{\mathrm{p}} \kappa \times\left(v_{\mathrm{s}}^{\mathrm{p}}-v_{\mathrm{L}}\right)$.

Here $\rho_{\mathrm{p}}$ and $n_{\mathrm{p}}=n_{\mathrm{e}}$ are the mass and number densities of the superconducting protons, $\kappa$ and $\boldsymbol{\Phi}_{0}$ are vectors directed along the flux line, with magnitudes $\kappa=h / 2 m_{\mathrm{p}}$ and $\Phi_{0}=h c / 2 e$, the quanta of vorticity and flux, respectively. From equations (5) and (6), one obtains the flux line velocity $v_{\Phi, r}$ in the direction away from the magnetic axis

$v_{\Phi, r}=\alpha\left(v_{\mathrm{s}}^{p}-v_{\mathrm{c}}\right)$,

where $\alpha$ is given by the ratio and inverse ratio of the inertial and drag coefficients:

$\alpha=\left[\frac{\rho_{\mathrm{p}} \kappa}{\eta}+\frac{\eta}{\rho_{\mathrm{p}} \kappa}\right]^{-1}=\left[\frac{n_{\mathrm{e}} e \Phi_{0}}{\eta c}+\frac{\eta c}{n_{\mathrm{e}} e \Phi_{0}}\right]^{-1}$.

The effective conductivity $\sigma_{\text {eff }}$ of Ruderman et al. (1998) can now be obtained in a quick and illuminating manner. The rotational (solenoidal) electric field $\mathbf{E}=-1 / c(\partial \mathbf{A} / \partial t)$ which plays the leading role in the dynamics of magnetic field decay (Passamonti et al. 2017) is set up by the radial flow of flux lines in the case of a Type II superconductor:

$E=-\frac{1}{c} \frac{\partial A}{\partial t}=\frac{n_{\Phi} \Phi_{0}}{c} v_{\Phi, r}$.

Using equation (7),

$E=\frac{\alpha n_{\Phi} \Phi_{0}}{n_{\mathrm{e}} e c} j \equiv \frac{j}{\sigma_{\mathrm{eff}}}$,

we obtain

$\sigma_{\mathrm{eff}}=\frac{n_{\mathrm{e}} e c}{\alpha n_{\Phi} \Phi_{0}}=\frac{n_{\mathrm{e}} e c}{n_{\Phi} \Phi_{0}}\left[\frac{n_{\mathrm{e}} e \Phi_{0}}{\eta c}+\frac{\eta c}{n_{\mathrm{e}} e \Phi_{0}}\right]$.

The expression for the drag coefficient $\eta$ on flux lines is scaled from the corresponding expression for the drag coefficient on magnetized vortex lines (Alpar et al. 1984b). The magnetized vortex line has a flux $\Phi_{*}$ and a London radius $\Lambda_{*}$ which depend on the superfluid proton flow dragged around the neutron vortex line, and therefore on the effective neutron and proton masses. For electron scattering from the flux line these quantities are $\Phi_{0}$ and the London radius $\Lambda$ for the proton superfluid, giving 
$\eta \cong 1.3 \times 10^{-2}\left(\rho_{\mathrm{p}, 14}\right)^{1 / 6} \rho_{\mathrm{p}} \kappa$,

where $\rho_{\mathrm{p}, 14}$ is the superconducting proton density in units of $10^{14} \mathrm{~g} \mathrm{~cm}^{-3}$. With $\rho_{\mathrm{p}} \kappa \gg \eta$, we obtain

$\sigma_{\mathrm{eff}}=\frac{\left(n_{\mathrm{e}} e\right)^{2}}{\eta n_{\Phi}}$.

Now we turn to the flux line-vortex line pinning. Flux lines (and vortex lines pinned to them) are moving with the velocity $v_{\mathrm{c}}+v_{\mathrm{L}, \mathrm{r}}$. To remain pinned, the neutron vortex lines would have to be moving at the velocity $v_{\mathrm{c}}+v_{\mathrm{L}, \mathrm{r}}-v_{\mathrm{S}}^{\mathrm{n}}$ with respect to the background neutron superfluid. According to the Magnus equation of motion such a nonzero velocity difference between vortex lines and background neutron superfluid is sustained by the pinning forces arising at flux-vortex junctions. Pinning cannot be sustained if the velocity difference exceeds the maximum (critical) velocity difference corresponding to the maximum pinning force available at the junction. The direction of $v_{\Phi, \mathrm{r}}$ is radially outward from the magnetic dipole axis, or outward from the circular symmetry axis of the toroidal distribution of flux lines, as the case may be. The $v_{\mathrm{c}}$ and $v_{\mathrm{s}}^{\mathrm{n}}$ are in the direction of the rotational flow, i.e. the azimuthal direction with respect to the rotation axis. Leaving directional aspects aside, pinning can be sustained, and so flux lines will move together with vortex lines in directions radially outward from the rotation axis, as the star spins down, if $v_{\Phi, \mathrm{r}} \lesssim v_{\mathrm{cr}}$. The critical velocity $v_{\mathrm{cr}}$ for a vortex line to remain pinned to a flux line, corresponding to the maximum pinning force per unit volume $F_{\max }$ is given by equation (4):

$v_{\mathrm{cr}} \cong \frac{F_{\max } c^{2}}{\sigma_{\mathrm{eff}}\left(n_{\Phi} \Phi_{0}\right)^{2}}$.

The pinning energy is determined by the magnetic energies in the cores of the flux lines and the spontaneously magnetized vortex lines. The magnetic cores of the flux and vortex lines carry fluxes $\Phi_{0}$ and $\Phi_{*}$ and have radii $\cong \Lambda$ and $\Lambda_{*}$ respectively. In the following estimations we will neglect factors $\sim O(1)$ that depend on effective nucleon masses, and adopt $\Phi_{*}=\Phi_{0}$ and $\Lambda_{*}=\Lambda$, the London penetration depth of the proton superconductor. For both superfluids $\Lambda>\xi_{i}$, the coherence length of the neutron or proton superfluid, and the magnetic energy in the flux line and magnetized neutron vortex line cores is larger than the condensation energy.

The number of flux line-vortex line junctions per unit volume is

$n_{\text {junc }}=\frac{\left(\pi \Lambda^{2}\right)^{2}}{l_{\Phi}^{2} l_{\text {vort }}^{2} V_{\text {junc }}}$, where $l_{\Phi}$ and $l_{\text {vort }}$ are the average spacings between flux and vortex lines respectively, and $V_{\text {junc }} \sim \Lambda^{3}$ is the volume of a flux-vortex junction; $l_{\Phi}{ }^{2}=\Phi_{0} / B$ and $l_{\text {vort }}{ }^{2}=2 \Omega / \kappa$. Note that $n_{\text {junc }}$ has the same dependence on the flux line and vortex line densities.

The pinning energy at each flux-vortex junction is estimated as

$E_{\text {pin }} \sim \frac{1}{8 \pi}\left(\frac{\Phi_{0}}{\pi \Lambda^{2}}\right)^{2} V_{\text {junc }} \ln (\Lambda / \xi)$.

The pinning energy is the magnetic energy gain in the overlap volume at the vortex line-flux line junction. The factor $\ln (\Lambda / \xi) \cong 5$ accounts for the local current structure around the flux and vortex lines. The maximum pinning force per junction is given by $F_{\text {pin }}=E_{\text {pin }} / \Lambda$ where $E_{\text {pin }}$ is the pinning energy at each flux-vortex junction. This leads to the maximum force per unit volume

$F_{\max }=F_{\text {pin }} n_{\text {junc }} \sim \frac{1}{8 \pi} \frac{\Phi_{0}^{2}}{l_{\Phi}^{2} l_{\text {vort }}^{2}} \frac{1}{\Lambda} \ln (\Lambda / \xi)$.

The result of Ruderman et al. (1998) is larger than this by a factor $l_{\Phi}^{2} / \Lambda^{2}$ because they take the spacing between the flux lines swept by a vortex line to which they are pinned to be $\sim \Lambda$, the minimum spacing allowed before the superconducting phase is lost at the higher critical field. Let me try to clarify this very high density of flux lines around the vortex line. Ruderman et al. (1998) wrote in the Appendix to their paper that 'the typical distance between two consecutive flux tubes pushed by the same moving vortex is about $\Lambda$... The magnetic repulsion between flux tubes limits their density. This repulsion is not effective until the inter-flux tube separation approaches $\Lambda$.' This enhanced density of flux lines carried along by a vortex line must be very local to the vicinity of the vortex line, which is so to speak, dressed by the cluster of pinned flux lines it has picked up during its entire journey through the star. The background configuration of the flux line array cannot have been effected. The extra energy cost of a non-uniform macroscopic average $B$ field would be prohibitively large if the pile up of flux lines plowed along by a vortex line extended to distances greater than $l_{\Phi}$. Thus as a vortex line moves the extra flux line density it carries along, it must be confined to distances of order $\Lambda$ in directions transverse to the vortex line. Other than this the flux line array between two vortex lines will be pushed forward by the vortex on one side and pulled along by the vortex on the front while retaining the equilibrium density $n_{\Phi}$, i.e. without being compressed. To check the consistency of this picture, we note that the vortex line needs to pick up $l_{\Phi} / \Lambda$ flux lines from each layer 
of the flux line array it encounters, corresponding to a fraction $l_{\Phi}^{2} / R \Lambda \sim 2 \times 10^{-14}$, taking the neutron star radius $R \sim 10^{6}, B \sim 10^{12} \mathrm{G}$ and $\Lambda \sim 100 \mathrm{fm}$. This small fraction of flux lines are those that are dislodged from the binding of the Abrikosov lattice, corresponding to the strong pinning regime described by Alpar et al. (1984a) in the context of vortex pinning in the neutron star crust lattice.

These considerations justify the estimate of Ruderman et al. (1998),

$F_{\max } \cong \frac{1}{8 \pi} \frac{\Phi_{0}^{2}}{l_{\text {vort }}^{2}} \frac{1}{\Lambda^{3}} \ln \left(\frac{\Lambda}{\xi}\right)$.

From equations (14) and (18), one obtains

$v_{\mathrm{cr}}=2 \times 10^{-10} \frac{\Omega}{B_{12}} \rho_{\mathrm{p}, 13}{ }^{2 / 3} \ln \left(\frac{\Lambda}{\xi}\right) \mathrm{cm} / \mathrm{s}$,

where $\rho_{\mathrm{p}, 13}$ is the density of superconducting protons in units of $10^{12} \mathrm{~g} \mathrm{~cm}^{-3}$.

For the steady state spin-down of the neutron superfluid at the rate $\dot{\Omega}$ dictated by the external torque, the average radial velocity of vortex lines at distance $r$ from the rotation axis is given by

$v_{\mathrm{vort}, r}=\frac{|\dot{\Omega}| r}{2 \Omega}$.

This average macroscopic velocity is due to all microscopic dissipative interactions between the normal matter core of the vortex line and ambient normal matter, e.g. electrons scattering from the vortex core. Flux lines and vortex lines will remain pinned and move together as long as $v_{\mathrm{vort}, r}$ remains less than the critical velocity, $v_{\mathrm{vort}, r}(r)<v_{\mathrm{cr}}$. This will hold at distances $r$ from the rotation axis satisfying

$r<r_{\mathrm{cr}} \cong 4 \times 10^{-10} \frac{\Omega^{2}}{|\dot{\Omega}| B_{12}} \rho_{\mathrm{p}, 13}{ }^{2 / 3} \ln \left(\frac{\Lambda}{\xi}\right) \mathrm{cm}$.

For pulsars older than the Vela pulsar (age greater than $10^{4}$ years) $r_{\mathrm{cr}} \gtrsim 10^{6} \mathrm{~cm}$, so that flux is being gradually expelled from the core of the neutron star. However, flux expelled from the core is not able to diffuse through the neutron star crust during the pulsar phase, as the magnetic field diffusion timescales through the high conductivity crust are estimated to be $\sim 10^{7} \mathrm{yr}$. Indeed the observed dipole moment distribution of young radio pulsars shows no indication of field decay.

At finite temperature vortex and flux lines will creep across pinning energy barriers by thermal activation. This process will allow the outward flow of vortex lines at the velocity $v_{\text {vort }, r}$ to achieve its steady state value, given in equations (20), defined by the external torque. This motion of the vortex lines in turn allows the superfluid to spin down at the steady state rate $\dot{\Omega}$. The velocity difference $v_{\infty}$ between $v_{\mathrm{c}}$ and $v_{\mathrm{s}}{ }^{\mathrm{n}}$ in steady state creep is always less than $v_{\text {cr }}$ so vortex creep will always operate when conditions allow pinning. Recent work interpreting certain components of post-glitch relaxation in the Vela pulsar and many older pulsars in terms of vortex creep against toroidal flux-lines (Gügercinoğlu \& Alpar 2014; Gügercinoğlu 2017) supports the conclusion that flux-vortex pinning and therefore flux decay induced by spin down in Srinivasan et al. (1990) do operate in these pulsars.

\section{Field decay under wind accretion}

Srinivasan et al. (1990) reasoned that the long term spindown of the neutron star by the companion's wind during the detached epoch of binary evolution is the decisive stage of evolution for the reduction of the average dipole magnetic field from $B \sim 10^{12} \mathrm{G}$ to $B \sim 10^{9} \mathrm{G}$ as the neutron star is spun down from $\Omega_{1} \sim 1$ $\operatorname{rad~s}{ }^{-1}$ when wind accretion starts at the end of the pulsar/ejector phase to $\Omega_{2} \sim 10^{-2}-10^{-3} \mathrm{rads}^{-1}$ as exemplified by systems like Vela X-1. Patruno et al. (2012) applied the scenario of Srinivasan et al. (1990) to the spin and magnetic field evolution of the accreting X-ray pulsar IGR J17480-2446 in Terzan 5. The neutron star was evolved through the wind accretion phase assuming $B(t) \propto \Omega(t)$ due to flux-vortex pinning.

After the pulsar crosses the death valley and pulsar activity stops, the neutron star continues to spin down under the dipole spindown torque until wind accretion starts when the Alfvén radius reaches the light cylinder. This happens at a rotation rate

$\Omega_{1}=6.4 \mu_{29}^{-4 / 7} \dot{M}_{11}^{2 / 7}\left(\frac{M}{1.4 M_{\odot}}\right)^{1 / 7} \operatorname{rad~s}^{-1}$,

where $\mu_{29}$ is the initial dipole moment of the neutron star, preserved through the initial radio pulsar phase, in units of $10^{29} \mathrm{G} \mathrm{cm}^{3}$ and $\dot{M}_{11}$ is the rate of mass capture by the neutron star from the wind in units of $10^{11} \mathrm{~g} \mathrm{~s}^{-1}$. The neutron star mass $M$ is given in units of 1.4 solar masses $M_{\odot}$. The wind mass loss rate of a solar mass main sequence star is about $10^{12} \mathrm{~g} \mathrm{~s}^{-1}$ and a few percent of this is expected to be captured by the neutron star (Nagae et al. 2004; Theuns et al. 1996), indicating mass accretion rates in the $10^{10}-10^{11} \mathrm{~g} \mathrm{~s}^{-1}$ range. The X-ray flux distribution of galactic neutron star binaries undergoing wind accretion (Pfahl et al. 2002) also indicates accretion rates in the $10^{11} \mathrm{~g} \mathrm{~s}^{-1}$ range. The wind accretion rate employed for IGR J17480-2446 
by Patruno et al. (2012) is two orders of magnitude larger in view of the large rotation rate expected for the synchronously rotating companion in that relatively young system. The LIGO discovery of gravitational radiation from the merging of two $\sim 30 M_{\odot}$ blackholes (Abbott et al. 2016a) implies weak winds for massive progenitor systems, especially in old, low metallicity populations (Abbott et al. 2016b). If weak winds are also common among few solar mass, main sequence companions especially with low metallicity, binaries with $\dot{M} \sim 10^{10} \mathrm{~g} \mathrm{~s}^{-1}$ may be common in old population environments which host the progenitors of LMXB and millisecond pulsars.

The wind accretion onto the neutron star produces a spin-down rate

$\dot{\Omega}=4.9 \times 10^{-17} I_{45}{ }^{-1} \dot{M}_{11} \mu_{29}{ }^{2 / 7}\left(\frac{M}{1.4 M_{\odot}}\right)^{3 / 7} \operatorname{rad~s}^{-2}$,

where $I_{45}$ is the moment of inertia of the neutron star in units of $10^{45} \mathrm{~g} \mathrm{~cm}^{2}$. Substituting these values in equation (21) one can check for consistency:

$$
\begin{aligned}
r_{\mathrm{cr}} \cong & 6.6 \times 10^{7} \mu_{29}{ }^{-10 / 7} \dot{M}_{11}^{-3 / 7}\left(\frac{M}{1.4 M_{\odot}}\right)^{-1 / 7} I_{45} \\
& \times\left(\rho_{p, 12}\right)^{2 / 3} \ln \left(\frac{\Lambda}{\xi}\right) \mathrm{cm} .
\end{aligned}
$$

Thus throughout the wind accretion era, $r_{\mathrm{cr}}$ is larger than the neutron star radius, so that flux vortex pinning prevails and spin-down induces field decay.

Thus the neutron star dipole magnetic moment will be reduced through the mechanism of Srinivasan et al. (1990), as the star spins down by wind accretion. Solving equation (23) with equation (1) leads to the spindown and field decay timescale

$$
\begin{aligned}
t_{\mathrm{sd}} & =\frac{7}{5} \frac{I \Omega_{1}}{\mu^{2 / 7} \dot{M}^{6 / 7}(G M)^{3 / 7}} \\
& \cong 6 \times 10^{9} \mu_{29}{ }^{-6 / 7} \dot{M}_{11}^{-4 / 7}\left(\frac{M}{1.4 M_{\odot}}\right)^{-2 / 7} \mathrm{yr} .
\end{aligned}
$$

\section{Conclusion}

The very simple and elegant mechanism proposed by Srinivasan et al. (1990) explains the comparatively weak magnetic dipole moments of millisecond pulsars by spin-down induced flux decay due to flux line-vortex line pinning. The mechanism will work during the long wind accretion phase of binary evolution. This is the crucial evolutionary phase for explaining the 1000-fold reduction in magnetic moments from the young radio pulsars to the old populations of LMXB, accreting $\mathrm{X}$-ray millisecond pulsars and millisecond radio pulsars. Thus their mechanism indeed produces the weak fields needed for the final LMXB spin-up to millisecond periods.

\section{Acknowledgements}

The author would like to wish G. Srinivasan a very happy 75th birthday and congratulate and thank him for his many brilliant contributions. The author first met G. Srinivasan in 1973 when he joined the Theory of Condensed Matter Group at the Cavendish Laboratory as a Ph.D. student of Phil Anderson and was charmed by his bright ideas and bright smile. The author also thanks Erbil Gügercinoğlu for his careful reading and suggestions and Onur Akbal for help with the manuscript.

\section{References}

Abbott, B. P. et al. 2016a, PhRvL, 116, 061102.

Abbott, B. P. et al. 2016b ApJ, 818, L22.

Alpar, M. A. 2008, A new class of radio pulsars - back in 1982, in: A Decade of Accreting Millisecond X-ray Pulsars (AIP Conference Proceedings), edited by R. Wijnands, D. Altamirano \& P. Soleri, vol. 1068, pp. 3-8.

Alpar, M. A., Cheng, A. F., Ruderman, M. A., Shaham, J. 1982, Nature, 300, 728.

Alpar, M. A., Anderson, P. W., Pines, D., Shaham, J. 1984a, ApJ, 276, 325.

Alpar, M. A., Langer, S. A., Sauls, J. A. 1984b, ApJ, 282, 533.

Backer, D. C., Kulkarni, S. R., Heiles, C., Davis, M. M., Goss, W. M. 1982, Nature, 300, 615.

Backer D. C., Kulkarni, S. R., Taylor, J. H. 1983, Nature, 301, 314.

Baym, G., Pethick, C. J., Pines, D. 1969, Nature, 224, 673.

Gügercinoğlu, E. 2017, MNRAS, 469, 2313.

Gügercinoğlu, E., Alpar, M. A. 2014, ApJ, 788, L11.

Muslimov, A. G., Tsygan, A. I. 1985, Ap\&SS, 115, 43.

Nagae, T. et al. 2004, A\&A, 419, 335.

Papitto, A. et al. 2013, Nature, 501, 517.

Patruno, A., Alpar, M. A., van der Klis, M., van den Heuvel, E. P. J. 2012, ApJ, 752, 33.

Passamonti, A, Akgün, T, Pons, J, Miralles, J. A. 2017, arXiv:1704.02016 [astro-ph.HE], accepted by MNRAS.

Pfahl, E., Rappaport, S., Podsiadlowski, P. 2002, ApJ, 571, L37.

Radhakrishnan, V., Srinivasan, G. 1982, Curr. Sci., 51, 1096. Ruderman, M. A., Zhu, T., Chen, K. 1998, ApJ 492, 267. 
Sauls, J. A. 1989, in: Timing Neutron Stars, NATO ASI Series $C, 262$, edited by H. Ogelman \& E. P. J. van den Heuvel, Kluwer, Dordrecht, p. 457.

Sidery, T., Alpar, M. A. 2009, MNRAS, 400, 1859.

Srinivasan, G. 1989, Astron Astrophys Rev., 1, 209.
Srinivasan, G., Bhattacharya, D. , Muslimov, A. G., Tsygan, A. I. 1990, Curr. Sci., 59, 31.

Theuns, T., Bofn, H. M. J., Jorissen, A. 1996, MNRAS, 280, 1264.

Wijnands, R., van der Klis, M. 1998, Nature, 394, 344. 